\title{
The Influence of Alfvén Ionization on Exoplanetary Atmospheres
}

\author{
Craig R. Stark ${ }^{1}$, Christiane Helling ${ }^{1}$ and Declan A. Diver ${ }^{2}$ \\ ${ }^{1}$ SUPA, School of Physics and Astronomy, University of St Andrews, \\ St Andrews, KY16 9SS, UK \\ email: craig.stark@st-andrews.ac.uk \\ ${ }^{2}$ SUPA, School of Physics and Astronomy, University of Glasgow, \\ Glasgow, G12 8QQ, UK
}

\begin{abstract}
Observations of radio emission from exoplanets and brown dwarfs suggest that such objects harbour atmospheric magnetized plasmas. However, the degree of thermal ionization for such substellar objects is insufficient to qualify the ionized component as a plasma, posing the question: what ionization processes can efficiently produce the required plasma? We propose Alfvén ionization as a mechanism for producing localized pockets of ionized gas in the atmosphere, having sufficient degrees of ionization $\left(\geqslant 10^{-7}\right)$ that they constitute plasmas. We outline the criteria required for Alfvén ionization to occur and justify its applicability in substellar atmospheres. For the model atmospheres considered here, our results show that degrees of ionization ranging from $10^{-6}-1$ can be obtained.
\end{abstract}

Keywords. plasmas, stars: atmospheres, stars: low-mass, brown dwarfs

\section{Alfvén Ionization}

Ultracool dwarfs (low-mass objects with spectral type later than M7; such as Brown Dwarfs, BDs, and gas giant planets, GPs) are observed to be strong sources of coherent radio emission (e.g. see Route \& Wolszczan 2012 and Hallinan et al. 2007 ), inferring the presence of atmospheric plasmas. We propose Alfvén ionization as a mechanism to produce the required plasma that is the source of the radiation.

In Alfvén ionization a constant stream of neutral gas impinges on a low-density magnetized plasma. The inflowing neutral atoms collide with and displace the plasma ions, leaving behind a significant charge imbalance that accelerates electrons to energies sufficient to ionize the local gas via electron-neutral impact ionization. Alfvén ionization requires: (i) an initial, low-density magnetized seed plasma; and (ii) a neutral gas flow that reaches a critical threshold speed (Alfvén 1960). The critical speed is obtained by equating the kinetic energy of the flow to the electrostatic potential energy of an electron in an electric potential equal to the ionization potential of a neutral gas particle $\phi_{I}$ : $\frac{1}{2} m_{\text {gas }} v_{c}^{2}=e \phi_{I}$. Therefore, the critical neutral gas flow speed is $v_{c}=\left(2 e \phi_{I} / m_{\text {gas }}\right)^{1 / 2}$. Species such as Potassium $\left(v_{c}=4.63 \mathrm{kms}^{-1}\right)$ have the smallest critical speeds whereas Hydrogen $\left(v_{c}=51.02 \mathrm{kms}^{-1}\right)$ and Helium $\left(34.43 \mathrm{kms}^{-1}\right)$ have the largest speeds. In substellar atmospheres the required seed plasma can be easily generated from local discharge events (lightning) in mineral clouds (Helling et al. 2011, 2013) and cosmic ray bombardment (Rimmer et al. 2013). Furthermore, typical magnetic flux densities of GPs $(\approx O(10 \mathrm{G}))$ can ensure that the seed plasma is sufficiently magnetized. 


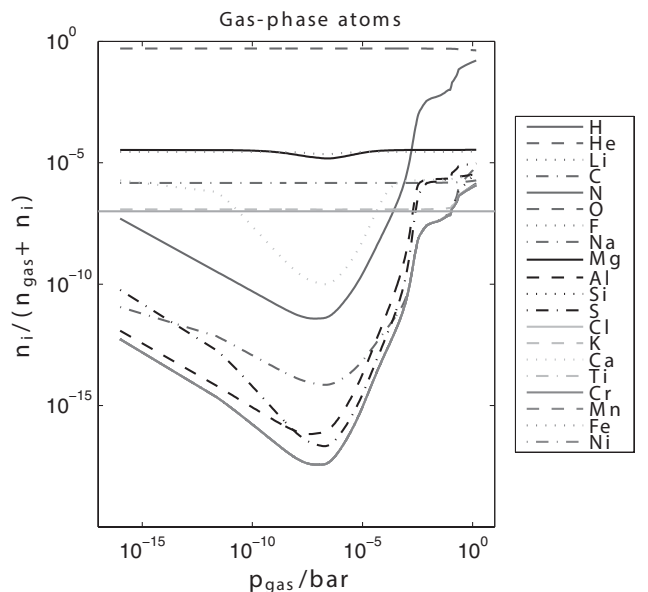

(a)

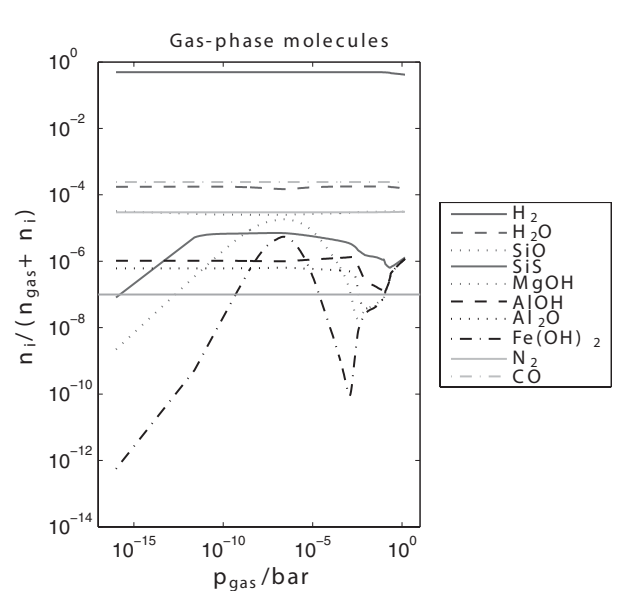

(b)

Figure 1. The degree of Alfvén ionization for a GP atmosphere. The grey horizontal line signifies the degree of ionization $\left(10^{-7}\right)$ required to constitute a plasma.

\section{Degree of Alfvén Ionization in exoplanetary atmospheres}

We are interested in Alfvén ionization in GP and BD atmospheres. GPs are similar objects to BDs but form differently and have a weaker magnetic field. Without loss of generality, we consider an example GP atmosphere using DRIFT-Phoenix which describes non-equilibrium cloud formation in substellar envelopes (Woitke \& Helling 2003, Helling et al. 2008, Helling et al. 2008b, Witte et al. 2009, Witte et al. 2011) characterized by $\log g=3.0, T_{\text {eff }}=1500 \mathrm{~K}$, for solar metallicity. Assuming conditions (i) and (ii) can be met, Alfvén ionization can ionize the entirety of the gas in a localized volume, leaving a plasma with an electron number density equal to the gas component number density (assuming $100 \%$ ionization) plus the initial seed magnetized plasma number density (Stark et al. 2013). Figs. 1(a) and 1(b) show the resulting degree of Alfvén ionization, if specific individual species constituting the atmospheric gas are entirely ionized (on their own) in a localized volume. In general, if in a localized atmospheric pocket a particular species can be $100 \%$ ionized, then the species with the greatest number density will yield the highest degree of ionization. To summarise: if entirely ionized on their own $\mathrm{He}, \mathrm{Fe}, \mathrm{Mg}$, $\mathrm{Na}, \mathrm{H}_{2}, \mathrm{CO}, \mathrm{H}_{2} \mathrm{O}, \mathrm{N}_{2}$ and $\mathrm{SiO}$ all consistently increase the degree of ionization beyond $10^{-7}$ throughout the model atmosphere considered here.

\section{References}

Alfvén, H. 1960, Rev. Mod. Phys., 32, 4, 710

Hallinan, G., et al. 2007, ApJ, 663, L25-L28

Helling, Ch., Jardine, M., Witte, S., \& Diver, D. A. 2011, ApJ, 727, 4

Helling Ch., Jardine, M., Stark, C. R., \& Diver, D. A. 2013, ApJ, 767, 136.

Helling, Ch., et al. 2008, MNRAS, 391, 1854

Helling, Ch., Woitke, P., \& Thi, W.-F 2008b, A\& A, 485, 547

Rimmer, P. B. \& Helling, Ch. 2013, ApJ, 774, 108

Route, M. \& Wolszczan, A. 2012, ApJ, 747, L22

Stark, C. R., et al. 2013, ApJ, 776, 11

Witte, S., Helling, Ch., Hauschildt, P. H. 2009, A\&A, 506, 1367.

Witte, S., Helling, Ch., Barman, T., Heidrich, N., Hauschildt, \& P. H. 2011, A\& A, 529, A44.

Woitke, P. \& Helling, Ch. 2003, A\&A, 399, 297 